\title{
Relato de experiência do funcionamento do programa de puericultura pelo curso de enfermagem na Faculdade Guaraí-FAG
}

O termo puericultura configura-se como informação referente aos cuidados no processo de evolução e crescimento da criança e para acompanhar esse desenvolvimento foi instituído o Programa de Puericultura, que visa acompanhar o crescimento do indivíduo, orientando quanto à cobertura vacinal, estimulando a prática do aleitamento materno, orientando quanto à alimentação complementar e prevenindo as complicações comuns nos bebes no decorre do dezoitos primeiros meses. Objetivo geral em relatar a experiência quanto ao funcionamento do programa de puericultura acompanhado pelos acadêmicos do curso de enfermagem na Faculdade Guaraí. A metodologia utilizada envolve a análise das fichas de acompanhamento às crianças, bem como relato das atividades desenvolvidas durante o acompanhamento, desde a sua implantação, fundamentando os aspectos apresentados por meio de publicações referentes ao tema. Conclui-se que as experiências dissertadas evidenciam que o programa atende satisfatoriamente à comunidade e contribuem para que os acadêmicos aperfeiçoem seus conhecimentos teóricos prático, ressaltando o compromisso da instituição quanto ao comprometimento da formação acadêmica.

Palavras-chave: Atendimento; Saúde pública; Qualidade.

\section{Experience report of the functioning of the child care program through the nursing course at the Guarai Faculty- FAG}

\begin{abstract}
The term childcare is configured as information referring to care in the child's evolution and growth process and to accompany this development, the Childcare Program was instituted, which aims to monitor the individual's growth, guiding the vaccination coverage, encouraging the practice of breastfeeding maternal, advising on complementary feeding and preventing complications common in babies during the first eighteen months. General objective in reporting the experience regarding the functioning of the childcare program accompanied by academics from the nursing course at Faculdade Guaraí. The methodology used involves the analysis of the follow-up sheets for children, as well as the report of the activities developed during the follow-up, since its implementation, supporting the aspects presented through publications related to the theme. It is concluded that the experiences presented show that the program satisfactorily serves the community and contribute for the academics to improve their theoretical and practical knowledge, highlighting the institution's commitment to the commitment of academic training.

Keywords: Service; Public health; Quality.
\end{abstract}

Barbara Núbia Silva Rocha Faculdade Guaraí, Brasil http://lattes.cnpq.br/6860511188584078 barbaranubia@hotmail.com

Brian Souza Cruz da Mota

Faculdade Guaraí, Brasil

http://lattes.cnpq.br/9191123594320775

brianmota@hotmail.com

Giullia Bianca Ferraciolli do Couto (iD

Faculdade Guaraí, Brasil

http://lattes.cnpq.br/0109560699727614

http://orcid.org/0000-0002-9768-778X

giulliabianca@hotmail.com
Juliane Marcelino dos Santos (iD)

Faculdade Guaraí, Brasil

http://lattes.cnpq.br/8751832120632358

http://orcid.org/0000-0002-2059-1069

julianemarcelino@hotmail.com

Adriana Keila Dias (iD

Faculdade Guaraí, Brasil

http://lattes.cnpq.br/2128882976477548

http://orcid.org/0000-0003-1291-5593

adrianakeiladias@hotmail.com

Reobbe Aguiar Pereira

Faculdade Guaraí, Brasil

http://lattes.cnpq.br/7447115724350334

http://orcid.org/0000-0003-2578-2611

enfreobbe@gmail.com
Glaucya Wanderley Santos Markus

Faculdade Guaraí, Brasil

http://lattes.cnpq.br/5568510365985231 http://orcid.org/0000-0001-8916-1086 glaucyamarkus@outlook.com
Referencing this:

ROCHA, B. N. S.; MOTA, B. S. C.; COUTO, G. B. F.; SANTOS, J. M.; DIAS, A. K.; PEREIRA, R. A.; MARKUS, G. W. S.. Relato de experiência do funcionamento do programa de puericultura pelo curso de enfermagem na Faculdade Guaraí-FAG. Scire Salutis, v.11, n.2, p.102108. 2021. DOI: http://doi.org/10.6008/CBPC2236$\underline{9600.2021 .002 .0011}$ 


\section{INTRODUÇÃO}

A enfermagem é uma profissão regulamentada pela Lei no 7.498/86 de 25 de junho de 1986 que descreve no seu Art. 2o que a Assistência apenas devem ser executadas por indivíduo inteiramente capacitado e escritas no órgão que regulamenta a profissão. Contempla ainda no Artigo 6으, inciso 1 que são enfermeiros o portador do documento de bacharel em enfermagem concedido por estabelecimento de ensino, conforme publicado na da lei (BRASIL, 2003).

A história nos mostra que as atividades relacionadas ao cuidado surgiram no Brasil por meio da Ana Neri e nos outros países por intermédio de Florence Nightingale. Após a organização da Enfermagem na Sociedade Brasileira por volta do final do século XIX, esta foi reconhecida como área de saúde, comprometida na prestação dos cuidados realizados antes, durante e após qualquer procedimento à vida humana. Assim, a atividade de enfermagem é livre em todo território nacional, podendo ser observada as disposições contempladas na lei no 7.498/1986 (BRASIL, 2002).

Nesse contexto, os cursos de graduação em enfermagem têm como objetivo durante a formação, a preparação de indivíduos íntegros e eficientes, aptos de colaborar para a promoção do bem-estar e da e das particularidades de existência dos cidadãos, bem como incrementar as formas de evitar danos, colocando em evidencia, oportunizando condições para um cuidado especializado a saúde, de maneira individualizada ou em no âmbito das instituições de saúde (BRASIL, 2003).

As incessantes mudanças nas normas de saúde exigem que a graduação dos acadêmicos de enfermagem seja um processo evolutivo da atuação profissional. Para que isto ocorra, as aulas práticas e os estágios supervisionados auxiliam diretamente para que estes desenvolvam as competências teóricas e apresentem suas habilidades e atitudes práticas, gerando no acadêmico um sentimento de segurança, autonomia e confiabilidade para se tornar um profissional competente e atuar com responsabilidade no processo de trabalho (EVANGELISTA, et al., 2014).

Assim, as Clinicas Escolas (CE), é um modelo utilizado em todo o mundo, que tem como objetivo ofertar um aprendizado significativo e aproximar o acadêmico da realidade que será submetido, após sua formação, essas, se destacam pela oportunidade de proporcionar aos acadêmicos momentos de interação com a realidade do processo de atendimento, pois possibilita o treinamento dos alunos, com aplicabilidade prática dos conhecimentos teóricos adquiridos no contexto de sala de aula, bem como contribui para o exercício da função social, pois atende à demanda da comunidade (EVANGELISTA, et al., 2014).

Diante desse contexto, a Clínica de Educação Para Saúde (CEPS/ Espaço Saúde), constituída por iniciativa do Instituto Educacional Santa Catarina - Faculdade de Guaraí (IESC-FAG), fundada em 2012, visa atender toda a população que necessita de assistência à saúde nas áreas de pediatria, enfermagem e fisioterapia, atendendo e desenvolvendo atividades que objetivam a promoção e prevenção em saúde, proporcionando assim uma melhora na qualidade de vida dos seus assistidos.

A Faculdade Guaraí realiza atendimento a comunidade por meio da CEPS, onde os acadêmicos do curso de enfermagem realizam o acompanhamento de puericultura, por meio das consultas de enfermagem, que constitui como um cuidado preventivo, sendo uma das vertentes da atenção à saúde da 
criança, por meio de orientação e promoção de ações que visem o bem-estar infantil e oportuno o tratamento em tempo hábil (GUIMARÃES, 2009).

O termo puericultura refere-se aos cuidados prestados ao recém-nascido até os dez anos de idade, podendo ser realizado em casa ou instituições de saúde, consiste em uma ferramenta oportuna para realização de educação em saúde e o acompanhamento de forma integral ao crescimento e desenvolvimento infantil (GUIMARÃES, 2009; BRASIL, 2012).

A puericultura tem um importante papel no sucesso do crescimento e desenvolvimento da criança, pois essa proporciona um acompanhamento individual, intimista, integral, holístico e humanista. Por essa razão obter uma boa adesão a essas consultas é imprescindível para a confirmação de seu sucesso, diante disso é possível afirmar que o Programa de Puericultura do curso de enfermagem, realizado na CEPS do IESC-FAG, atende um número satisfatório de criança de 0 aos 24 meses de vida? Essas consultas ocorrem de forma integral e assídua?

O presente artigo se justifica pela oportunidade de esclarecer a importância do programa de puericultura, bem como sua contribuição quanto a experiência para os acadêmicos, a fim de que esses aperfeiçoem seus conhecimentos e ainda possa oportunizar um acompanhamento sistematizado às crianças.

O objetivo geral proposto nesse artigo pretende relatar as experiências do funcionamento do programa de puericultura desenvolvido na CEPS do IESC/FAG. E como objetivos específicos descrever o conceito e as ações de enfermagem nas consultas de puericultura; narrar o processo de planejamento e organização das consultas de puericulturas na CEPS/espaço saúde e apresentar e discutir dados do seu primeiro ano de implantação.

\section{METODOLOGIA}

Este artigo trata-se de um relato das experiências dos acadêmicos do curso de enfermagem, sobre os atendimentos de puericultura na CEPS do IESC, este refere às vivencias de um estágio curricular e analise de Ficha Ambulatorial de Enfermagem (FAE). É importante considerar que a metodologia utilizada tem caráter descritivo, quantitativo e exploratório uma vez que o tipo de pesquisa tem como objetivo favorecer ampla intimidade com a dificuldade, para torná-la mais explícita ou constituir novas hipóteses.

Para analisar a efetividade do programa de puericultura na CEPS avaliou-se as FAEs do mês de implementação e consequentemente dos 11 meses posteriores, ou seja, de junho de 2017 a julho de 2018, tornando-se então o critério para uma FAE ser analisada. Em relação aos dados, foram analisados sexo, faixa etária e número de consultas mensais de crianças de 0 a 24 meses de vida.

Para embasamento teórico envolveu-se pesquisa bibliográfica permitindo uma relação próxima com autores que discorrem do tema. A busca foi feita em fonte que possuíam informações de caráter cientifico, sendo eles a Biblioteca Virtual em Saúde (BVS), por meio da BIREME (Centro Latino-Americano e do Caribe de Informação em Ciências da Saúde), nas Bases Eletrônicas Medline (Medical Literature Analysis and Retrietal System On-Line), Lilacs (Literatura Latino-Americana e do Caribe em Ciências da Saúde), 
SCiELO (Scientific Eletronic Library On Line). Será utilizado ainda como fonte de pesquisa o Caderno de Atenção Básica, no 33, do Ministério da Saúde, Secretaria de Atenção à Saúde, Departamento de Atenção Básica bem como as informações dos instrumentos utilizados no atendimento de puericultura.

\section{RESULTADOS E DISCUSSÃO}

As consultas de puericultura têm como calendário o acompanhamento da criança a cada dois meses, durante as mesmas os pais recebem orientações quando aos primeiros cuidados com o recémnascido, sua higiene corporal, cuidados com o coto umbilical, amamentação e introdução alimentar, cuidados com a pele, primeira dentição, vacinação, avaliação e informações quanto ao crescimento e desenvolvimento da criança e apoio aos pais nos primeiros dois anos de vida do filho, este é realizado pelo docente supervisor de estágio, os acadêmicos no $5^{\circ}$ período de enfermagem que cursam a disciplina de assistência de enfermagem a criança e ao adolescente I e os estagiários do $7^{\circ}$ e $9^{\circ}$ período de enfermagem na disciplina de estágio curricular $I$.

Os gráficos apresentados demonstram os atendimentos realizados desde a implantação do projeto de extensão das consultas de enfermagem em Puericultura em julho de 2017 a junho de 2018.

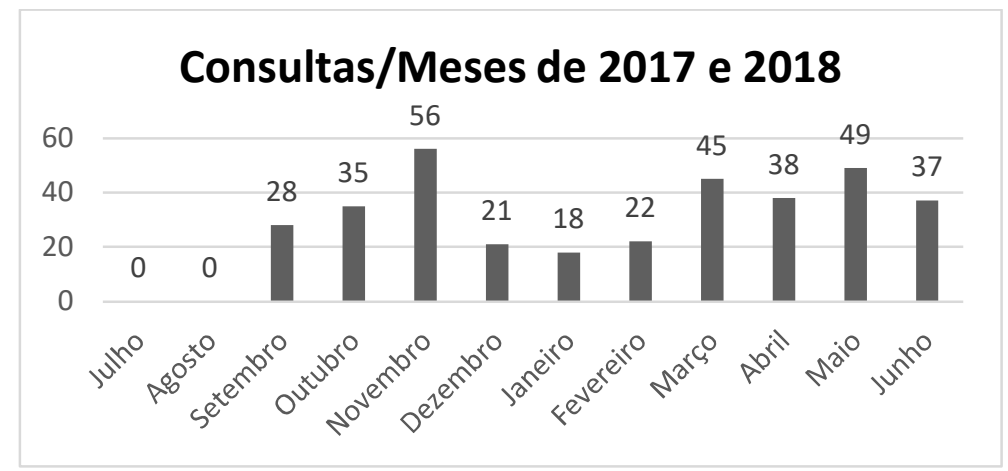

Gráfico 01: Atendimentos no período de julho 2017 a junho 2018.

No gráfico supracitado, visualizam-se os atendimentos realizados no mês de julho do ano de 2017 a junho de 2018. Nos meses de julho e agosto de 2017 não houve atendimentos. O mês de novembro de 2017 apresentou o mês com maior número de pacientes atendidos. Os meses seguintes, ou seja, dezembro do ano de 2017 ao mês de fevereiro de 2018 obtivemos menor número de atendimentos em relação aos meses seguintes. Nos meses de março a junho de 2018 tivemos poucas alterações em relação aos números de consultas de puericultura.

Nos meses de julho e agosto não foi registrado nenhum atendimento, considerando que o programa estava em processo de implantação e, por tanto foi o período de divulgação e ajustes do mesmo. Desta maneira o programa ainda estava em processo de construção pelo docente supervisor e discente da enfermagem, este era pouco conhecido e não havia uma busca ativa dos pacientes.

Diante desse contexto, o processo de implantação entendido como ação de instaurar, de iniciar uma atividade ou ainda instituição de um serviço de atributo singular geram situações peculiares que comprometem a logística e consequentemente dificultam a atuação da equipe (CIAMPO et al., 2009).

Nos meses de setembro e outubro o número de atendimentos foi significativo, demonstrando sua 
aceitação e qualidade do serviço, neste momento foi possível notar a importância da comunicação direta, paciente e paciente, a busca ativa nas Unidades Básicas de Saúde, por meio dos agentes comunitários de saúde, enfermeiros da Estratégia Saúde da Família e equipe de saúde do Hospital Regional de Guaraí. Por meio dessas equipes foram repassadas informações sobre o funcionamento da CESP e os atendimentos realizados, o que elevou o número de atendimentos.

Assim, a divulgação do projeto foi extremamente importante, considerando que essa ação oportuniza esclarecimento aos cooperadores quanto à estruturação das atividades que precisam ser desenvolvidas, como também busca instituir uma atitude colaborativa dos envolvidos em uma relação de proximidade com a comunidade, formando vínculos que favorecem a rotina das atividades desenvolvidas e reduzindo as situações de desconfortos geralmente ocasionadas pela falta de conhecimento (EVANGELISTA et al., 2014).

Quanto à competência acadêmica percebe-se que quanto maior for o envolvimento do aluno no processo de atendimento, mais eficaz será a sua atuação frente ao atendimento realizado. As situações de aprendizagem têm total relevância e nutrem a estruturação dos saberes dos educandos no gerenciamento da aprendizagem.

Assim, a escola deve ser concebida como ambiente de busca e constituição de conhecimentos complementados com as práticas sociais, produzindo significado ao saber aprendido. Todo esse processo qualifica o acadêmico quanto a sua participação no mundo (BRASIL, 2008). Na análise do gráfico II estão expostos os números de consultas realizadas conforme a idade dos pacientes atendidos.

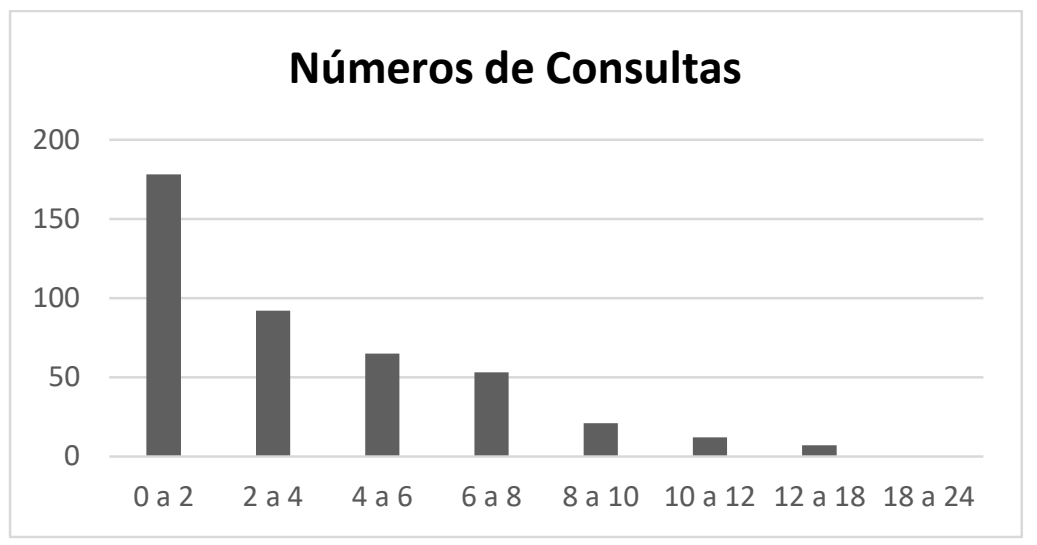

Gráfico 02: Atendimentos por faixa etária.

O número de lactentes atendidos de 0 a 02 meses de idade obteve um número expressivo de atendimentos, de 02 a 06 meses houve uma redução significativa nas consultas de puericultura. A partir dos 8 meses os números diminuem drasticamente, em comparação as consultas realizadas nos primeiros meses de vida da criança.

As consultas ao lactente de 0 a 02 meses de vida tiveram um número bem maior em relação às demais consultas, essa diferença pode estar ligada a inexperiência e inseguranças dos pais e a busca constante por informações e orientações que ajudem nos primeiros cuidados ao recém-nascido. Este demonstrou também que as consultas de puericultura foram iniciadas na época adequada, isto é, antes da criança completar 1 mês de idade. 
O desempenho do enfermeiro como agente de promoção do cuidado, se define como essencial no início da vida, auxiliando o recém-nascido, na sua adequação extrauterina, de cognição dos pais, quanto os amparando no processo de confrontação diante do novo contexto de vida e de todos os desafios interpostos (CRUZ et al., 2007).

Durante esta consulta, são realizados o acolhimento aos pais e a criança e a aferição dos sinais vitais. Os pais são orientados quanto à identificação de problemas, tais como vômitos, regurgitações que podem evoluir para um quadro de refluxo, secreções oculares por alteração nos ductos lacrimais, a exposição do lactente ao sol, nos horários de sol mais brando e não deve exceder a 30 minutos, estímulos adequados para idade, lavagem de nariz, higiene geral, curativo do coto umbilical, e avaliado crescimento e desenvolvimento do lactente (ALENCAR, 2012; BRASIL, 2011).

Outra informação repassada é quanto ao resultado do Teste do Pezinho, que no caso de alteração o médico deverá ser informado para que o procedimento seja realizado o mais precocemente, permitindo dessa forma que os agravos sejam minimizados com o tratamento adequado.

Nas consultas de 2 a 4 meses, a busca de informações por parte dos pais ainda é uma realidade, considerando os números de atendimento realizados. Esse período é considerado como a fase das descobertas e os pais buscam saber se seu filho está apresentando conforme normalidade, ressaltando a confiança no profissional, a busca por acompanhamento e especialmente a pretensão de instruir-se.

Nas consultas de 4 a 6 meses, observa-se uma diminuição, justificado pela não observância de problemas aparentes em relação à saúde do bebê. Contudo, apesar da aparência saudável pode se ter a falsa ideia de que a criança não precisa de acompanhamento, o que é um engano, considerando que o objetivo do acompanhamento de puericultura é justamente a prevenção para que o bebê cresça cada vez mais saudável.

No período de vida entre 2 a 4 meses, além dos procedimentos de rotina a mãe é orientada quanto ao método da ordenha para a coleta do leite, caso a mãe trabalhe fora, é reforçado ainda quanto ao direito de desconto de uma hora no período de trabalho para o aleitamento, que pode ser dirimida em dois intervalos de trinta minutos cada. A mãe deve ser aconselhada a amamentar antes de retirar-se do seu lar e após a sua chegada (ALENCAR, 2012).

Assim, conhecer a criança de forma holística, permite que os pais observem cada situação adversa causada por inúmeras condições, sejam elas quanto ao desenvolvimento físico, psicológico, alimentar, considerando que a partir dos seis meses outros costumes são inseridos ao contexto de desenvolvimento e a criança, como também os pais precisam se adaptar (MORAES, et al., 2008).

As consultas de 8 aos 10 meses houve uma queda no número de atendimentos quando comparado aos meses anteriores, ligadas principalmente a segurança dos pais, considerando que a criança está se desenvolvendo dentro dos padrões de normalidade e a aceitação da dieta. Durante essas consultas são realizadas a aferição dos sinais vitais, avaliação do aumento da criança, seguidas de orientações quanto à introdução alimentar, sono e repouso e estímulos ao desenvolvimento (TOSCANO et al., 2003).

Dados referentes ao atendimento por sexo não foram demonstrados, ressaltando que não obteve 
diferença significativa. O atendimento às pacientes do sexo feminino alcançou uma média de 15,5 representando cerca de $9,7 \%$ e aos pacientes do sexo masculino foram em média de 14,4 representando 9,4\%. Isso demonstra que há uma equiparação quanto aos números de crianças atendidas indiferente do sexo.

É pertinente ressaltar que o responsável por realizar as consultas de puericultura na maioria são as mães e, portanto, a diferença de sexo não influencia nas orientações, considerando que as informações repassadas durante as consultas observando as fases do desenvolvimento da criança, não há divergência quanto ao sexo, pois ambos obedecem ao mesmo critério de desenvolvimento.

\section{CONCLUSÕES}

O estudo nos permitiu contatar que o acompanhamento ao desenvolvimento da criança durante as consultas de puericultura é de fato essencial e tem como objetivo, dentre outros, o agenciamento, amparo e a constatação antecipada de alterações passíveis de mudança que repercutem de forma negativa na vida futura do recém-nascido. As atividades educativas como as informações repassadas em cada atendimento se constituem como fundamentais, considerando que permite à família sanar dúvidas quanto as mais diversas situações, admitindo um acompanhamento sistematizado e integral à saúde da criança (BRASIL, 2008).

Assim, de modo geral os resultados apresentados foram satisfatório, mesmo ficando aquem do que se estabelece como ideal, quando se refere ao acompanhamento do lactente conforme determina as orientações do programa de puericultura do Ministério da Saúde.

Diante desse fato, o desafio para a enfermagem fica evidenciado, considerando que esse profissional precisa estruturar melhor suas estratégias para alcançar o objetivo deste e de outros programas, para que a comunidade possa enxergar que o cuidado prestado pelos profissionais de saúde não se realiza apenas no âmbito da cura, mais principalmente no sentido de previnir e fazer com que os sujeitos se percebam como responsável pela promoção de sua saúde.

Assim é pertinente destacar que o trabalho desenvolvido pela CESP, ainda é embrionário e precisa de ajustes, mas que já tem refletido consideravelmente tanto na prática educativa quanto para a comunidade, pois proporciona aos acadêmicos a oportunidade de vivenciar situações da vida real e consequentemente a resolução dos possíveis problemas que envolvem a comunidade.

Ressalta-se, no entanto, que é importante realizar a avaliação do atendimento prestado por meio da análise gradativa dos dados, pois permite que as ações sejam implementadas e dessa forma atenda aos princípios definidos pela Constituição Federal, ou seja, que visem principalmente à equidade e ao progresso das situações de vida para a comunidade.

Diante do que foi abordado, ajuizar na saúde o bebê é pensar em um acompanhamento que deve ser realizado considerando sua estrutura física, motora e intelectual, visto que é um indivíduo que está em constante desenvolvimento e, portanto necessita ser cuidado para que tenha uma vida saudável.

Assim, as ações para que haja uma maior adesão da comunidade nos programas envolve vários 
aspectos dentre os quais estão: as políticas públicas como fundamento da proposta de desenvolvimento humano, melhor divulgação quanto à importância dos programas, capacitação de recursos humanos principalmente quanto às orientações de como e onde realizar as consultas de puericultura, dando continuidade ao cuidado iniciado no pré-natal. Dessa forma, o esforço contínuo dos profissionais com as famílias das crianças, deve ser de transmitir segurança e envolvê-lo no processo de cuidado, visto que são corresponsáveis no contexto saúde doença. A elaboração de protocolos e estruturação de estratégias de qualidade, incorporado ao esforço conjunto da equipe gestora e dos profissionais se configura como tática de qualidade dentro de uma visão integrada da saúde, considerando o acolhimento como princípio básico da humanização do atendimento.

\section{REFERÊNCIAS}

ALENCAR, C. P.. Programa de qualidade da empresa Ceará Diesel - Mercedes-Benz: um estudo de caso. 2012.

BRASIL. Indagações sobre currículo: educandos e educadores: seus direitos e o currículo. Brasília: Ministério da Educação, 2008.

BRASIL. Acompanhamento do crescimento e desenvolvimento infantil. Brasília: Ministério da Saúde, 2002.

BRASIL. Dez passos para uma alimentação saudável: Guia alimentar para crianças menores de 2 anos. Brasília: Ministério da Saúde, 2003.

BRASIL. Orientações para a coleta e análise de dados antropométricos em serviços de saúde: norma técnica do Sistema de Vigilância Alimentar e Nutricional - SISVAN. Brasília: Ministério da Saúde, 2011.

BRASIL. Profissionalização de auxiliares de enfermagem: cadernos do aluno: fundamentos de enfermagem. 2 ed. Brasília: Ministério da Saúde, 2003.

BRASIL. Saúde da criança: crescimento e desenvolvimento infantil. Brasília: Ministério da Saúde, 2012.
CRUZ, D. C. S; SUMAM, N. S; SPÍNDOLA, T.. Os cuidados imediatos prestados ao recém-nascido e a promoção do vínculo mãe-bebê. Ver. Esc. Enfermagem USP, v.41, n.4, p.690-97, 2007.

CIAMPO, L. A; RICCO, R. G.; DANELUZZI, J. C; CIAMPO, I. R. L; FERRAZ, I. S; ALMEIDA, C. A. N.. Puericultura: uma prioridade a ser resgatada. Jornal de Pediatria, v.16, n.4, p.158-61, 2009.

EVANGELISTA, D. L; IVO, O. P.. Contribuições do Estágio Supervisionado para a formação do profissional de enfermagem: expectativas e desafios. Revista Enfermagem Contemporânea, v.3, p.123-130, 2014.

GUIMARÃES, S. S.. Psicologia da saúde e doenças crônicas. In: KERBAUY, R. R.. Comportamento e saúde: explorando alternativas. Santo André: ArtBytes, 2009

MORAES, J. C; RIBEIRO, M. C. S. A.. Desigualdades sociais e cobertura vacinal: uso de inquéritos domiciliares. Rev. Bras. Epidemiol, v.11, n.1, p.13-124, 2008.

TOSCANO, C.; KOSIM, L.. Cartilha de vacinas: para quem quer mesmo saber das coisas. Brasília: Organização PanAmericana da Saúde, 2003.

A CBPC - Companhia Brasileira de Produção Científica (CNPJ: 11.221.422/0001-03) detém os direitos materiais desta publicação. Os direitos referem-se à publicação do trabalho em qualquer parte do mundo, incluindo os direitos às renovações, expansões e disseminações da contribuição, bem como outros direitos subsidiários. Todos os trabalhos publicados eletronicamente poderão posteriormente ser publicados em coletâneas impressas sob coordenação da Sustenere Publishing, da Companhia Brasileira de Produção Científica e seus parceiros autorizados. Os (as) autores (as) preservam os direitos autorais, mas não têm permissão para a publicação da contribuição em outro meio, impresso ou digital, em português ou em tradução. 